\title{
Effect of ankle range of motion modification on dynamic valgus index during a monopodal squat in female soccer players \\ Efecto de la modificación del rango de movimiento del tobillo sobre el índice de valgo dinámico durante una sentadilla monopodal, en jugadoras de fútbol \\ *Andrés Felipe Flores-León, **Vania Constanza Leyton Quezada, ***María José Martínez Hernández, ***Daniela Almendra Salazar Reinoso, ****José Francisco Berral de la Rosa \\ *Universidad de los Andes (Chile), **ARC Sport Medicine (Chile), ***Clínica Dávila (Chile), **** Universidad Pablo de Olavide (España)
}

\begin{abstract}
The purpose of this study was to determine the effect of simulated ankle range of motion modification on the dynamic valgus index (DVI) of female soccer players during a monopodal squat. A second objective compared the frontal plane projection angles (FPPA) of hip and knee to identify the greatest contribution to the DVI.The dominant leg (DL) and non-dominant leg (NDL) were evaluated in 16 female soccer players during a monopodal squat, under Basal conditions: «without wedge» (WW); Restrictive: «with dorsiflexor wedge» (DW) and Facilitator: «with plantiflexor wedge» (PW) of ankle. With the Kinovea software, the DVI was identified by adding the hip and knee FPPA. The three conditions in the dominant leg and non-dominant leg, the FPPA in each condition were compared and effect sizes were determined. No condition showed differences in DVI in DL vs. NDL. In DL, DVI increased with large effect in DW and decreased with large effect in PW compared to WW and DW. In NDL, DVI increased with large effect in DW compared to WW and PW. In both legs, hip FPPA was higher than knee FPPA in all conditions. It is concluded that the simulated restriction of ankle dorsiflexion is effective for an increase in DVI, while the facilitator allows its decrease with greater effect on NDL. The hip is the joint with the greatest contribution to the DVI, independent of condition.
\end{abstract}

Key words: Dynamic knee valgus, ankle dorsiflexion, ankle plantiflexion, wedge, two-dimensional kinematics.

Resumen. El propósito de este estudio fue determinar el efecto de la modificación simulada del rango de movimiento del tobillo sobre el índice de valgo dinámico (IVD) de jugadoras de fútbol, durante una sentadilla monopodal. Un segundo objetivo comparó los ángulos de proyección en el plano frontal (APPF) de cadera y rodilla para identificar el mayor aporte al IVD. Se evaluó en 16 mujeres futbolistas, durante una sentadilla monopodal, la pierna dominante (PD) y no dominante (PND), en las condiciones Basal: «sin cuña (SC); Restrictiva: «con cuña dorsiflexora» (CD) y Facilitadora: «con cuña plantiflexora» (CP) de tobillo. Con el software Kinovea se identificó el IVD sumando los APPF de cadera y rodilla. Se compararon las tres condiciones en la pierna dominante y no dominante, los APPF en cada condición y se determinaron los tamaños de efecto. Ninguna condición presentó diferencias en IVD en PD vs PND. En PD, el IVD aumentó con gran efecto en CD y disminuyo con gran efecto en CP comparado con SC y CD. En PND, el IVD aumentó con gran efecto en CD comparado con SC y CP. En ambas piernas, el APPF de cadera fue mayor que el de rodilla en todas las condiciones. Se concluye que la restricción simulada de la dorsiflexión de tobillo es efectiva para un aumento del IVD, mientras que la facilitadora permite su disminución con mayor efecto en PND. La cadera es la articulación con mayor aporte al IVD, independiente de la condición.

Palabras claves:Valgo dinámico de rodilla, dorsiflexión de tobillo, plantiflexión de tobillo, cuña, cinemática bidimensional.

\section{Introduction}

During soccer practice, it has been recognized that women present a $5.7 \%$ higher probability of suffering injuries compared to men (Faude et al., 2005). More than two-thirds of all injuries occur mostly in the lower limb of players, with knee (27\%) and ankle (22\%) injuries being the most common (Dick et al., 2007; Faude

Fecha recepción: 30-09-21. Fecha de aceptación: 27-01-22 Andres Felipe Flores-Leon

aflores@uandes.cl et al., 2005). These injuries commonly occur without contact, affecting the supporting leg of soccer players (Brophy et al., 2010).

The movement performed by the player when kicking the ball requires body support and joint control of the knee in a monopodal stance (Chew-Bullock et al., 2012). While performing the movement, the supporting leg must resist the reaction forces of the ground by reducing the speed of the hip, which slows the body during the kick, thus stabilizing the action (Lees et al., 2010). It has been identified that misalignment of the foot and hip of the supporting leg in female soccer 
players is a predictor of frontal plane knee misalignment (Lees et al., 2010). In addition to the demands of the change of speed and direction generated in this sport, greater knee joint stability is required in order to reduce the risk of injury (Bloomfield et al., 2007; Feria et al., 2015; Viviescas et al., 2021).

The risk of knee injury has been associated with dynamic valgus, defined as the medial displacement of the knee in the frontal plane during functional activity (Mauntel et al., 2013). This movement is associated with mechanisms of injury prevalent in female athletes, such as anterior cruciate ligament injury (Hewett et al., 2005). To assess knee dynamic valgus, the monopodal squat has been used as a test that replicates the demand and injury risk of the lower limb in athletes (Bailey et al., 2011; Ludwig et al., 2017; Munro et al., 2017; Ugalde et al., 2015; Wyndow et al., 2016). The clinical evaluation of the dynamic valgus has been shown to have acceptable reliability, which increases when complemented by kinematic evaluation (Nae et al., 2017; Ugalde et al., 2015). The 2D capture systems are highly reliable in evaluating the monopodal squat and have minimal variability with respect to $3 \mathrm{D}$ analysis (Gwynne \& Curran, 2014). Scholtes \& Salsich (2017), determined the dynamic valgus index (DVI) as the sum of the frontal plane projection angles (FPPA) of the hip and knee (Scholtes \& Salsich, 2017). This index has a higher intraclass correlation coefficient than the detection of the FPPA of the knee, when assessing the dynamic valgus of this joint during a functional task (Scholtes \& Salsich, 2017).

Increased knee dynamic valgus has been associated with a reduced ankle dorsiflexion angle, both of which are associated with increased risk of lower limb injury (Barra et al., 2021; Lima et al., 2018). This association has also been observed in female soccer players during the landing of a vertical jump (Malloy et al., 2015; Sigward et al., 2008). Previous studies have analyzed the effects of the initial ankle position on knee dynamic valgus during functional tasks in healthy individuals (Mohd Azhar et al., 2019; Pangan \& Leineweber, 2021; Tran et al., 2016). Macrum et al. (2012), used a plantar wedge with forefoot lift to simulate the decrease in ankle dorsiflexion range during a bilateral squat (Macrum et al., 2012). The results of this research determined that the initial position in ankle dorsiflexion produced an increase in knee dynamic valgus (Macrum et al., 2012). On the contrary, the heel lift or the foot lift in decline produces a reduction in knee dynamic valgus during the bilateral squat, due to the initial plantiflexion position that facilitates the ankle dorsiflexion ROM (Bell et al., 2013; Bell-Jenje et al., 2016). However, there are no studies that analyze the magnitude of the change that occurs in the control of the frontal plane of the support leg of female soccer players, when both the gain and loss of ankle range of motion are simulated, in the lateral plane. This information can be useful to know the kinematic adaptations of the lower limb of the soccer player in conditions that replicate loss or increase in ankle dorsiflexion, facilitating the use of the wedge as a rehabilitation strategy and prevention of prevalent injuries in these athletes.

The purpose of the present research was to determine the effect of simulated ankle range of motion modification on DVI in female soccer players during a monopodal squat. At the same time, we sought to compare the knee and hip FPPA in each condition, in order to identify the greater contribution to the DVI that occurs in the dominant and non-dominant leg of the female athletes. Our hypothesis was that a simulated condition of ankle dorsiflexion restriction is effective in reproducing an increase in the DVI, while the facilitating condition is effective in decreasing this index, with the hip being the joint with the greatest contribution to the increase in the DVI of female soccer players.

\section{Materials and methods}

\section{Study design}

An observational, analytical and cross-sectional study design was used.

\section{Sample description}

The sample was calculated using GPower 3.1 software, using data obtained from the study by Bell-Jenje et al. (2015). The variable hip adduction range (dynamic knee valgus) during the execution of a monopodal squat was analyzed with values of $18.3^{\circ} \pm 5.9^{\circ}$ and $11.8^{\circ} \pm$ $4.1^{\circ}$ in the groups with and without ankle dorsiflexion deficits, respectively (Bell-Jenje et al., 2016). Subsequently, the difference of the average values was calculated, obtaining a value of $6.5^{\circ} \pm 1.8^{\circ}$. Thus, an effect size of 3.61; considering a power of $95 \%$ and a significance level of .05 , two-tailed. Thus, the sample size calculation for this study identified a minimum value of four volunteers. However, this study evaluated a soccer team of 20 players.

The inclusion criteria corresponded to female soccer players from a professional team, between 18 and 35 years of age, who practice soccer weekly and who have 
a BMI between 18.5 - $29.9 \mathrm{~kg} / \mathrm{m}^{2}$ (Ruiz et al., 2019). Athletes were excluded if they reported having a lower limb injury within the last six months that limited the performance of the test, a history of surgery or current pain in the lower limbs or the lumbopelvic segment, and those who presented a limitation of the ankle dorsiflexion angle greater than $10 \%$ in relation to the contralateral limb.

The final sample consisted of 16 female players aged $22 \pm 5$ years, weighing $59.34 \pm 6.63 \mathrm{~kg}$, with a height of $1.60 \pm .07 \mathrm{~m}$, and a BMI of $23.10 \pm 2.37 \mathrm{~kg} / \mathrm{m}^{2}$; who did not present ankle dorsiflexion angle limitation and who reported an average of 10 hours of practice and 1.5 hours of competitive play weekly. The dominant side was observed in the left leg in one player, while 15 showed dominance of the right leg.

\section{Participant recruitment}

The sample was chosen by convenience. In the first instance, a member of the medical team of the sports club was contacted and a day was arranged to visit the training site. The female soccer players were invited to participate voluntarily and independently of the suggestions of the coach or members of the medical team. A brief selection survey was carried out in order to identify the inclusion and exclusion criteria of the sample. All participants read the informed consent form and then signed it freely, agreeing to participate in the research. This consent is based on the 2013 Declaration of Helsinki, which was previously approved by the local scientific ethical committee.

\section{Evaluation protocol}

At the beginning of the evaluations, personal data, basic measurements of demographic variables, anthropometric measurements and the dorsiflexion range of motion (ROM) of each player were recorded in the evaluation form.

For the anthropometric measurement, each participant had to stand on a scale and measuring rod (SECA 700, Hamburg, Germany) to measure their weight $(\mathrm{kg})$ and height $(\mathrm{cm})$, in order to subsequently calculate the BMI. These evaluations were performed in anatomical position. The data were handled individually according to each athlete and were the exclusive knowledge of the evaluators, respecting the confidentiality of the participants.

The ankle dorsiflexion ROM was evaluated with the athlete standing barefoot in front of a wall, with the purpose of corroborating the symmetry between extremities. This evaluation was based on a previous protocol (Hoch \& McKeon, 2011). The tibia-wall angle (TWA) was measured using a goniometer, positioning the fulcrum on the lateral condyle of the knee, the fixed arm parallel to the wall and the movable arm in the direction of the lateral malleolus of the ankle. The ankle dorsiflexion ROM was calculated using the dorsiflexion angle with the following formula: DFA $=180-$ (TWA $+90)$. The limitation of this angle was considered to be the loss of more than $10 \%$ in relation to the contralateral limb, which was considered as an exclusion criterion.

Subsequently, the identification of the dominant leg was performed, where each volunteer was thrown a ball that she had to catch with her foot, freely, at a distance of three meters. This test was performed three times and was supervised by another evaluator. The dominant leg was defined as the leg she used the most when performing this task. The result of this evaluation was corroborated by the participant's response to the question about their skilled side, which was recorded on the evaluation card. This research assessed the monopodal or supported condition of the dominant and non-dominant leg. A warm-up based on active joint mobility was performed before the start of the tests.

\section{Kinematic evaluation of tests}

Prior to the kinematic evaluation, the participant had to dress in tight black clothing, which was provided by the research team to facilitate the contrast in the recording records. Over this clothing, each participant was provided with reflective markers 20 millimeters wide. Markers were placed bilaterally on the greater trochanter, lateral femoral condyle, lateral face of peroneal malleolus, the anterior superior iliac spines, midpoint of the femoral condyles and midpoint of the ankle malleoli.

Tests were performed with and without the use of a plantar wedge in both limbs. The wedge was constructed with dimensions of $40 \mathrm{~cm}$ long, $8.5 \mathrm{~cm}$ high, $15 \mathrm{~cm}$ wide and an angle of $12^{\circ}$ (Macrum et al., 2012). The soccer player was requested to perform the monopodal squat in conditions that modified the position of the ankle: the basal condition was performed on the ground and was nominated as «without wedge» (WW); the simulated condition of ankle dorsiflexion restriction was performed on the wedge that caused forefoot lift and was nominated «with dorsiflexor wedge» (DW); while the facilitating condition of the dorsiflexion path was executed with the wedge lifting the heel and was nominated «with plantiflexor wedge» (PW) (Figure 1). 


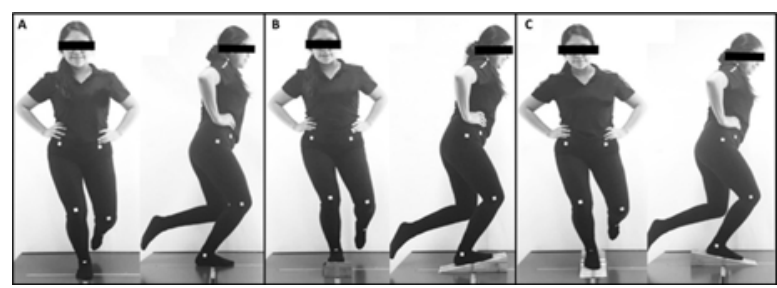

Figure 1. Execution of the monopodal squat.

A: Basal condition: without wedge $(W W) \cdot B \cdot$ Ankle motion restrictive condition: with dorsiflexor plantar

wedge (DW); C:Ankle motion facilitating condition: with plantiflexor plantar wedge (PW)

An evaluator instructed the correct execution of the monopodal squat by positioning the hands on the waist, keeping the trunk upright and the foot placed on previously demarcated lines both on the floor and on the wedge. Each test was executed following the timing of a metronome with a frequency of 60 pulses/minute that defined three seconds for the descent phase and one second for the ascent. Each volunteer was instructed to perform the descent until maximum knee flexion was achieved without lifting the heel. For each condition, three repetitions were recorded randomly, which were also combined in relation to the dominant and nondominant lower limb that was in support. This randomization was done to avoid the volunteers becoming used to the test. Thus, each player recorded a total of 36 recordings for analysis, where 18 corresponded to the sagittal plane and 18 to the frontal plane.

The kinematic analysis was performed using Kinovea software (version 0.8.15). First, the sagittal plane recording was analyzed. Markers located on the greater trochanter, lateral femoral condyle and lateral malleolus of the supporting leg were digitally attached to identify the knee flexion angle during the monopodal squat. Frame-by-frame analysis identified the millisecond in which the participant achieved $60^{\circ}$ of knee flexion in the descent phase. For this, the marker located on the lateral condyle of the femur was used as a fulcrum, then a straight line was drawn to the marker located on the lateral face of the greater trochanter and another projected towards the lateral face of the lateral malleolus (Figure 2.A). Once this data was obtained, the synchronized recording was analyzed in the frontal plane. The previously indicated millisecond was located and the markers of the anterior superior iliac spines and the anterior midpoint of the femoral condyles of the supporting leg were joined, determining the á angle. Thus, the hip FPPA was calculated as $90^{\circ}$-á. Subsequently, the markers of the iliac spine of the supporting leg, the anterior midpoint of the femoral condyles, and the anterior midpoint of the ankle malleoli were joined to identify the â-angle. Thus, the knee FPPA was calculated

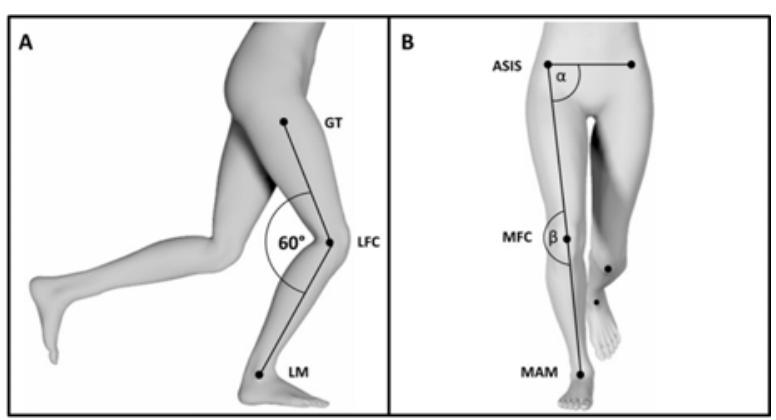

Figure 2. Schematic diagram of the kinematic analysis.

A: Identification of the $60^{\circ}$ of knee flexion as the point of analysis. B: Identification of the frontal plane projection angle of hip (a) and knee ( $(B)$. The schematic also shows the location of the markers in both planes: (Sagittal) GT: greater trochanter; LFC: lateral femoral condyle; LM: lateral malleolus; (Frontal) ASIS: anterior superior iliac spines; $M F C$ : midpoint of the femoral condyles; $M A M$ : midpoint of ankle malleoli.

as $180^{\circ}$ - $\hat{a}$ (Figure 2.B). The DVI was determined based on the following formula: DVI= Hip FPPA + knee FPPA.

\section{Statistical analysis}

The value for each participant corresponded to the average of the three repetitions for each condition. These data were grouped according to condition as WW, DW and PW of the dominant and non-dominant leg. The Shapiro-Wilk test was applied, which identified a parametric distribution of the data. Both the comparison of the condition in relation to the dominant and nondominant side, as well as the comparison of hip and knee FPPA angles in each condition, was performed using Student's t-test for repeated measures. A significance level of $p<.05$ was considered for all results. These statistical calculations were performed with PRISM 6 software (GraphPad Software, California, USA).

The effect size was determined using Cohen's d. Cohen has previously defined effect sizes as; small $(\mathrm{d}=.20)$, medium $(\mathrm{d}=.50)$ and large $(\mathrm{d}=.80)$ (Cohen, 1988). GPower software (V.3.1.9.2, University of Kiel, Germany) was used for these statistical calculations.

\section{Results}

The comparison of the DVI of the supporting leg during the execution of the monopodal squat on the dominant vs. non-dominant side, did not present significant differences in WW (dominant=35.96 \pm 9 . 66; non-dominant $=28.15 \pm 7.90 ; \mathrm{p}=.05 ; \mathrm{d}=.85)$, DW (dominant $=49.25 \pm 13.16$; non-dominant $=48.21 \pm$ $6.19 ; p=.77 ; \mathrm{d}=.09$ ) and $\mathrm{PW}($ dominant $=25.35 \pm 9.80$; non-dominant $\left.=25.29 \pm 8.57 ; p^{<.01 ;} \mathrm{d}=.98 ;\right)$. When comparing the DVI of the dominant leg during monopodal squat execution, $\mathrm{WW}$ is significantly lower than DW with a large effect size $\left(p^{<.01 ;} \mathrm{d}=1.14\right)$. While the DVI in WW is significantly greater than in 
PW with a large effect size $(p<.01 ; \mathrm{d}=1.09)$. The DVI in DW is significantly higher than in PW, with a large effect size $(p<.01 ; \mathrm{d}=2.01)$.

When comparing the DVI of the non-dominant leg during monopodal squat execution, WW is significantly lower than DW with a large effect size $(p<.01 ; \mathrm{d}=3.05)$. The DVI in WW presents no difference with the PW condition, the effect size being small $(p=.36 ; d=.40)$. While the DVI in DW is significantly higher than in PW, with a large effect size $(p<.01 ; \mathrm{d}=3.04)$.

The data and analyses of the conditions evaluated in the dominant and non-dominant leg are shown in Table 1 .

\begin{tabular}{|c|c|c|c|c|c|}
\hline & $\begin{array}{c}\text { Mean difference } \\
\text { (standard deviation) }\end{array}$ & $\begin{array}{l}95 \% \text { Confidence } \\
\text { interval }\end{array}$ & $p$ Value & Cohen's d & Effect size \\
\hline \multicolumn{6}{|l|}{ DVI dominant leg } \\
\hline WW vs. DW & $-13.29(2.73)$ & -20.38 to $-6,206$ & $<.01$ & 1.14 & Large \\
\hline WW vs. PW & $10.61(1.78)$ & 5.989 to 15.22 & $<.01$ & 1.09 & Large \\
\hline DW vs. PW & $23.90(2.41)$ & 17.64 to 30.15 & $<.01$ & 2.01 & Large \\
\hline \multicolumn{6}{|c|}{ DVI non-dominant leg } \\
\hline WW vs. DW & $-20.06(1.82)$ & -24.79 to -15.33 & $<.01$ & 3.05 & Large \\
\hline WW vs. PW & $2.87(2.02)$ & -2.381 to 8.093 & .36 & .40 & Small \\
\hline DW vs. PW & $22.92(1.98)$ & 17.76 to 28.07 & $<.01$ & 3.04 & Large \\
\hline
\end{tabular}

The comparison between FPPA evidenced that, in both the dominant and non-dominant leg, that of the hip is significantly greater than that of the knee in both WW (dominant: $p<.01, \mathrm{~d}=.92$; non-dominant: $p<.01$, $\mathrm{d}=.89$ ), DW (dominant: $p<.01, \mathrm{~d}=.96$; non-dominant: $p<.01, \mathrm{~d}=1.18$ ) and PW (dominant: $p<.01, \mathrm{~d}=1.32$; non-dominant: $p<.01, \mathrm{~d}=.96)$ conditions. The data are shown in Table 2.

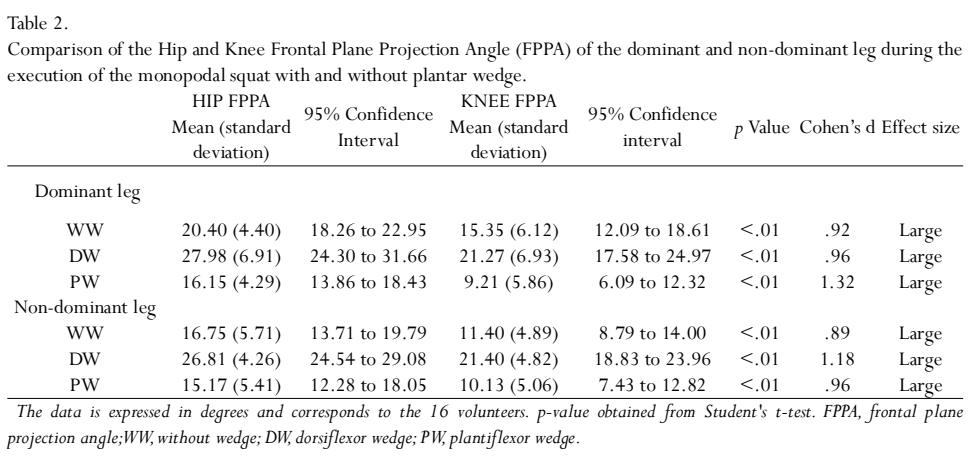

\section{Discussion}

The results obtained in the present study show that the DVI increases when a restrictive condition of ankle dorsiflexion is simulated and decreases when the condition facilitates this movement. These findings are similar to frontal plane biomechanical adaptations observed in people with lower extremity impairments (De Bleecker et al., 2020). The meta-analysis by Lima et al. (2018), describes that there is an association between reduced ankle dorsiflexion and knee dynamic valgus, proposing that preventive programs should address these conditions (Lima et al., 2018). Regarding knee alterations, it has been described that people with anterior cruciate ligament injury have a lower degree of ankle dorsiflexion compared to healthy people (Wahlstedt \& Rasmussen-Barr, 2015). It has also been observed that a low range of ankle dorsiflexion can generate a high risk of knee tendinopathy in athletes (Backman \& Danielson, 2011; Malliaras et al., 2006). Therefore, the controlled simulation of the loss of ankle dorsiflexion produced by a $12^{\circ}$ wedge could favor the training of frontal plane knee movement control in a demanding condition and therefore its use as an injury prevention strategy. For its part, the study by Freke et al. (2020), evaluated the influence of a $25^{\circ}$ decline plank on monopodal squat performance in hip arthroscopy candidates, identifying a reduction in medial knee displacement independent of the participants' hip pain. (Freke et al., 2020). The wedge in a declined position could be used as a mechanism to facilitate control of knee movement in the frontal plane, in those soccer players who present difficulty or greater risk of injury due to the presence of an increased DVI.

The simulated loss of ankle dorsiflexion (monopodal over DW squat), produced results similar to those reported by Macrum et al. (2012), who reproduced this condition with a wedge lifting both forefeet while performing a bilateral squat and compared it to the flat surface execution, observing increased knee dynamic valgus (Macrum et al., 2012). The restrictive condition of ankle dorsiflexion could be responsible for increased eversion of the rearfoot as a compensatory mechanism to unlock the joint, which is associated with medial knee displacement in the frontal plane (Barton et al., 2012; Petersen et al., 2014). The review by Petersen et al. (2017), describes that rearfoot eversion is one of the causes responsible for knee dynamic valgus associated with both athletes with patellofemoral pain and individuals with osteoarthritis of this joint (Petersen et al., 2017).

For its part, the simulated increase in ankle dorsiflexion range was effective in decreasing DVI. Zwerver et al. (2007) described that during a monopodal squat the ankle is in a dorsiflexion position, generating a lower knee moment and a greater sensation of calf tightness (Zwerver et al., 2007). Contrary to 
dorsiflexion, the position in ankle plantiflexion reduces the compensations of the rearfoot and ankle in the descent phase of the squat, causing a decrease in knee dynamic valgus (Bell et al., 2013). Previous studies mention that the execution of this task on a decline board also favors knee flexion range (Purdam et al., 2004; Young et al., 2005). Although the decline angle used by these studies was $25^{\circ}$, it has been reported that an angle greater than $16^{\circ}$ could increase gastrocnemius activity, resulting in a lower mechanical advantage during the execution of the monopodal squat (Richards et al., 2008). However, it has also been observed that a decline greater than $15^{\circ}$ increases the activity of the posterior leg and thigh muscles, which allows for knee stability in the sagittal plane during the bilateral squat (Richards et al., 2016). In the present study, the wedge angle was $12^{\circ}$, which facilitated the execution of the monopodal squat with at least $60^{\circ}$ of knee flexion in both conditions, finding similar effects to those reported with greater angles. This finding could be used when determining the degree of plantiflexion required to assess or facilitate frontal plane knee control.

On the other hand, the present work does not identify DVI differences between the dominant and nondominant leg. However, the effect size was large in comparison to squats performed on a flat surface or WW. The lowest DVI detected in the non-dominant leg in the basal condition presented a level like that of the facilitator condition (monopodal squat on PW), which could explain the similarities of this index in both conditions. This could be associated with the greater monopodal control that players have in their less skilled leg, which acts as a support during the kicking movement (Chew-Bullock et al., 2012).

A second objective compared knee and hip FPPAs to identify the angle most associated with increased DVI. From both the dominant and non-dominant leg of female athletes, the hip angle generates a greater effect on the DVI compared to the knee angle, even when the monopodal squat was performed in the facilitating condition. Sholtes \& Salshic (2017), identified that women with patellofemoral pain present a higher DVI than those who do not, but after verbal instruction they correct this alteration by decreasing hip and knee FPPA (Scholtes \& Salsich, 2017). Ho et al. (2021) compared the DVI and FPPA of the hip and knee in people with and without anterior cruciate ligament injury during a jump test, identifying that there is an increase in the DVI and knee FPPA in people with injury, but they mention that there was also a tendency to increase in the hip FPPA (Ho \& Murata, 2021). Although both studies identify that the increase in DVI is associated with an increase in FPPA, a situation that was also observed in this study, they did not analyze the possible differences between them. From our knowledge, this is the first study comparing hip and knee angles in the frontal plane in healthy female athletes. Modified simulation of ankle position would produce greater or lesser control of the knee in the frontal plane, with greater effect on the contribution of the hip angle. Rabin et al. (2016), identified that individuals with less ankle dorsiflexion increased hip adduction during a lateral squat (Rabin et al., 2016). It has also been observed that a monopodal squat with the ankle in decline improves lateral trunk inclination in people with hip weakness (Freke et al., 2020). Therefore, identifying the effects of the FPPAs that make up the DVI separately during simulated conditions would facilitate the detailed analysis of the involved joint during the evaluation and preventive management of lower limb injuries in female soccer players.

This study is not without limitations. First, the evaluation of ankle dorsiflexion range was only used as an inclusion criterion, so future reports could evaluate the range of limitation in the sagittal plane and its association with DVI. Because this study limited its evaluation to a group of healthy female soccer players, the results cannot be projected to men, so future research could compare both sexes. Finally, 2D motion capture methods may present errors during data evaluation and processing, however, this study attempted to reduce these variables based on the methodology presented.

The effect found in the present study allows us to elucidate that lower limb movement conditions associated with injury risk or efficient biomechanical corrections during functional movements can be reproduced. This may be useful when evaluating and training female soccer players to perform mechanically demanding tasks that simulate an athletic motion. The data obtained from this research may be of interest to professionals involved in exercise science, health, and sport, and those in charge of the preventive and therapeutic management of lower limb disorders.

\section{Conclusion}

The simulated condition of ankle dorsiflexion restriction is effective in reproducing the increased DVI of female soccer players, which has been associated with an increased risk of lower limb injury. Furthermore, 
the facilitating condition of the greater range of movement of the ankle dorsiflexion causes a positive effect for decreasing this range which is associated with greater control of movement, mainly when the supporting leg is the dominant one. The hip is the joint that contributes the most to the increase in DVI, independent of the position of the ankle or the dominant side of the athlete's supporting leg.

\section{Conflict of interest}

The authors declare no conflict of interest.

\section{Acknowledgments}

We would like to express our great appreciation to the people who voluntarily participated in this study.

\section{References}

Backman, L. J., \& Danielson, P. (2011). Low range of ankle dorsiflexion predisposes for patellar tendinopathy in junior elite basketball players: a 1-year prospective study. The American journal of sports medicine, 39(12), 2626-2633.

Bailey, R., Selfe, J., \& Richards, J. (2011). The single leg squat test in the assessment of musculoskeletal function: a review. Physiotherapy practice and research, 32(2), 18-23.

Barra, C. M. S. M., Cabezas, G. R., \& Gajardo, J.T. (2021). Propuesta de modelo predictivo de riesgo de lesión en base a descriptores anatómicos y funcionales que se relacionan con la inestabilidad articular en rodilla y tobillo en jugadores de baloncesto no profesionales. Retos: nuevas tendencias en educación fisica, deporte y recreación(39), 54.

Barton, C. J., Levinger, P., Crossley, K. M., Webster, K. E., \& Menz, H. B. (2012). The relationship between rearfoot, tibial and hip kinematics in individuals with patellofemoral pain syndrome. Clinical biomechanics, 27(7), 702-705.

Bell, D. R., Oates, D. C., Clark, M.A., \& Padua, D. A. (2013).Twoand 3-dimensional knee valgus are reduced after an exercise intervention in young adults with demonstrable valgus during squatting. Journal of athletic training, 48(4), 442-449.

Bell-Jenje, T., Olivier, B., Wood, W., Rogers, S., Green, A., \& McKinon, W. (2016). The association between loss of ankle dorsiflexion range of movement, and hip adduction and internal rotation during a step down test. Manual Theraphy, 21, 256261. https://doi.org/10.1016/j.math.2015.09.010

Bloomfield, J., Polman, R., \& O’Donoghue, P. (2007). Physical Demands of Different Positions in FA Premier League Soccer. InJ Sports Sci Med (Vol. 6, pp. 63-70).

Brophy, R., Silvers, H. J., Gonzales, T., \& Mandelbaum, B. R. (2010). Gender influences: the role of leg dominance in ACL injury among soccer players. British journal of sports medicine, 44(10), 694-697.

Chew-Bullock, T. S.,Anderson, D. I., Hamel, K.A., Gorelick, M.
L., Wallace, S.A., \& Sidaway, B. (2012). Kicking performance in relation to balance ability over the support leg. Human Movement Science, 31(6), 1615-1623. https://doi.org/10.1016/ j.humov.2012.07.001

Cohen, J. (1988). Statistical Power Analysis for the Behavioral Sciences (2nd Edition ed.). Lawrence Eearlbaum Associates.

De Bleecker, C., Vermeulen, S., De Blaiser, C., Willems, T., De Ridder, R., \& Roosen, P. (2020). Relationship Between JumpLanding Kinematics and Lower Extremity Overuse Injuries in Physically Active Populations: A Systematic Review and MetaAnalysis. Sports Medicine, 50(8), 1515-1532. https://doi.org/ 10.1007/s40279-020-01296-7

Dick, R., Putukian, M., Agel, J., Evans, T. A., \& Marshall, S.W. (2007). Descriptive Epidemiology of Collegiate Women's Soccer Injuries: National Collegiate Athletic Association Injury Surveillance System, 1988-1989Through 2002-2003. Journal of Athletic Training, 42(2), 278-285.

Faude, O., Junge, A., Kindermann, W., \& Dvorak, J. (2005). Injuries in female soccer players: a prospective study in the German national league. The American Journal of Sports Medicine, 33(11), 1694-1700. https://doi.org/10.1177/0363546505275011

Feria, A., De Hoyo, M., Romero, S., Mateo, J., \& Sañudo, B. (2015).Varo y valgo de rodilla en cambios de dirección como factor de riesgo de lesión. Retos: nuevas tendencias en educación fisica, deporte y recreación (26), 176-177. https: / /doi.org/https: / /recyt.fecyt.es/index.php/retos/article/view/34462

Freke, M. D., Crossley, K., Russell, T., Sims, K. J., \& Semciw, A. (2020). Changes in Knee andTrunk Alignment in PeopleWith Hip Pain and Healthy Controls When Using a Decline Board During Single-Leg Squat. Journal of Sport Rehabilitation, 29(7), 886-896.https://doi.org/10.1123/jsr.2019-0097

Gwynne, C. R., \& Curran, S.A. (2014). Quantifying frontal plane knee motion during single limb squats: reliability and validity of 2-dimensional measures. International Journal of Sports PhysicalTherapy, 9(7), 898-906.

Hewett, T. E., Myer, G. D., Ford, K. R., Heidt, R. S., Jr, Colosimo, A. J., McLean, S. G., van den Bogert, A. J., Paterno, M.V., \& Succop, P (2005). Biomechanical measures of neuromuscular control and valgus loading of the knee predict anterior cruciate ligament injury risk in female athletes: a prospective study. The American Journal of Sports Medicine, 33(4), 492-501. https: / / doi.org/10.1177/0363546504269591

Ho, K.-Y., \& Murata, A. (2021). Asymmetries in Dynamic Valgus Index After Anterior Cruciate Ligament Reconstruction: A Proof-of-Concept Study. International Journal of Environmental Research and Public Health, 18(13), 7047.

Hoch, M. C., \& McKeon, P. O. (2011). Normative range of weightbearing lunge test performance asymmetry in healthy adults. Manual Therapy, 16(5), 516-519. https://doi.org/10.1016/ j.math.2011.02.012

Lees, A., Asai, T., Andersen, T. B., Nunome, H., \& Sterzing, T. (2010). The biomechanics of kicking in soccer: A review. Journal of sports sciences, 28(8), 805-817.

Lima,Y. L., Ferreira, V., de Paula Lima, P. O., Bezerra, M. A., de Oliveira, R. R., \& Almeida, G. P. L. (2018).The association of ankle dorsiflexion and dynamic knee valgus: A systematic review and meta-analysis. Physical Therapy in Sport, 29, 61-69. 
https://doi.org/10.1016/j.ptsp.2017.07.003

Ludwig, O., Simon, S., Piret, J., Becker, S., \& Marschall, F. (2017). Differences in the dominant and non-dominant knee valgus angle in junior elite and amateur soccer players after unilateral landing. Sports, 5(1), 14.

Macrum, E., Bell, D. R., Boling, M., Lewek, M., \& Padua, D. (2012). Effect of limiting ankle-dorsiflexion range of motion on lower extremity kinematics and muscle-activation patterns during a squat. Journal of Sport Rehabilitation, 21(2), 144-150.

Malliaras, P., Cook, J. L., \& Kent, P. (2006). Reduced ankle dorsiflexion range may increase the risk of patellar tendon injury among volleyball players. Journal of science and medicine in sport, 9(4), 304-309.

Malloy, P., Morgan,A., Meinerz, C., Geiser, C., \& Kipp, K. (2015). The association of dorsiflexion flexibility on knee kinematics and kinetics during a drop vertical jump in healthy female athletes. Knee Surgery, Sports Traumatology, Arthroscopy, 23(12), 3550-3555.

Mauntel, T. C., Begalle, R. L., Cram, T. R., Frank, B. S., Hirth, C. J., Blackburn, T., \& Padua, D. A. (2013). The effects of lower extremity muscle activation and passive range of motion on single leg squat performance. The Journal of Strength \& Conditioning Research, 27(7), 1813-1823. https://doi.org/ 10.1519/JSC.0b013e318276b886

Mohd Azhar, N., Affandi, N. F., Mail, M. S. Z., \& Shaharudin, S. (2019). The effects of foot position on lower extremity kinematics during single leg squat among adolescent male athletes. Journal of Taibah University Medical Sciences, 14(4), 343349. https: / /doi.org/10.1016/j.jtumed.2019.06.007

Munro, A., Herrington, L., \& Comfort, P. (2017).The relationship between 2-dimensional knee-valgus angles during single-leg squat, single-leg-land, and drop-jump screening tests. Journal of sport rehabilitation, 26(1), 72-77.

Nae, J., Creaby, M. W., Cronstrom, A., \& Ageberg, E. (2017). Measurement properties of visual rating of postural orientation errors of the lower extremity - A systematic review and metaanalysis. PhysicalTherapy in Sport, 27, 52-64.https: / /doi.org/ 10.1016/j.ptsp.2017.04.003

Pangan,A. M., \& Leineweber, M. (2021). Footwear and Elevated Heel Influence on Barbell Back Squat: A Review. Journal of Biomechanical Engineering, 143(9). https://doi.org/10.1115/ 1.4050820

Petersen, W., Ellermann, A., Gösele-Koppenburg, A., Best, R., Rembitzki, I. V., Brüggemann, G. P., \& Liebau, C. (2014). Patellofemoral pain syndrome. Knee Surgery, SportsTraumatology, Arthroscopy, 22(10), 2264-2274. https: / / doi.org/10.1007/ s00167-013-2759-6

Petersen,W., Rembitzki, I., \& Liebau, C. (2017). Patellofemoral pain in athletes. Sports Medicine, 8, 143-154. https://doi.org/ 10.2147/oajsm.s133406

Purdam, C. R., Jonsson, P., Alfredson, H., Lorentzon, R., Cook, J. L., \& Khan, K. M. (2004). A pilot study of the eccentric decline squat in the management of painful chronic patellar tendinopathy. British Journal of Sports Medicine, 38(4), 395-397. https: / /doi.org/10.1136/bjsm.2003.000053

Rabin,A., Portnoy, S., \& Kozol, Z. (2016). The association of ankle dorsiflexion range of motion with hip and knee kinematics during the lateral step-down test. Journal of orthopaedic \&sports physical therapy, 46(11), 1002-1009.

Richards, J., Selfe, J., Sinclair, J., May, K., \& Thomas, G. (2016). The effect of different decline angles on the biomechanics of double limb squats and the implications to clinical and training practice. Journal of human kinetics, 52, 125-138. https:// doi.org/10.1515/hukin-2015-0200

Richards, J., Thewlis, D., Selfe, J., Cunningham, A., \& Hayes, C. (2008). A biomechanical investigation of a single-limb squat: implications for lower extremity rehabilitation exercise. Journal of Athletic Training, 43(5), 477-482. https: / /doi.org/10.4085/ 1062-6050-43.5.477

Ruíz, A. C., Legleu, C. E. C., de León Fierro, L. G., Luján, R. C., \& Rodríguez, B. O. (2019). Somatotipo de mujeres futbolistas universitarias por posición en el terreno de juego. Retos:nuevas tendencias en educación física, deporte y recreación(36), 228-230.

Scholtes, S.A., \& Salsich, G. B. (2017). A dynamic valgus index that combines hip and knee angles: assessment of utility in females with patellofemoral pain. International Journal of Sports Physical Therapy 12(3), 333-340.

Sigward, S. M., Ota, S., \& Powers, C. M. (2008). Predictors of frontal plane knee excursion during a drop land in young female soccer players. Journal of orthopaedic \& sports physical therapy, 38(11), 661-667.

Tran, A. A., Gatewood, C., Harris, A. H., Thompson, J. A., \& Dragoo, J. L. (2016). The effect of foot landing position on biomechanical risk factors associated with anterior cruciate ligament injury. Journal of Experimental Orthopaedics, 3(1), 13. https: / /doi.org/10.1186/s40634-016-0049-1

Ugalde,V., Brockman, C., Bailowitz, Z., \& Pollard, C. D. (2015). Single leg squat test and its relationship to dynamic knee valgus and injury risk screening. PM\&R, 7(3), 229-235; quiz 235.https:/ /doi.org/10.1016/j.pmrj.2014.08.361

Viviescas, A. M. A., Pinzón, D. M. N., de Sousa, H. C. D., Moreno, J. D. E., Medina, D. B., \& Delgado, J. C. S. (2021). Sprint pattern analysis of professional female soccer players on artificial and natural turf. Retos: nuevas tendencias en educación física, deporte y recreación(39), 97.

Wahlstedt, C., \& Rasmussen-Barr, E. (2015). Anterior cruciate ligament injury and ankle dorsiflexion. Knee Surgery, Sports Traumatology,Arthroscopy, 23(11), 3202-3207.https://doi.org/ 10.1007/s00167-014-3123-1

Wyndow, N., De Jong,A., Rial, K., Tucker, K., Collins, N.,Vicenzino, B. , Russell, T., \& Crossley, K. (2016). The relationship of foot and ankle mobility to the frontal plane projection angle in asymptomatic adults. Journal of Foot and Ankle Research, 9, 3. https: / /doi.org/10.1186/s13047-016-0134-9

Young, M.A., Cook, J. L., Purdam, C. R., Kiss, Z. S., \& Alfredson, H. (2005). Eccentric decline squat protocol offers superior results at 12 months compared with traditional eccentric protocol for patellar tendinopathy in volleyball players. British journal of sports medicine, 39(2), 102-105.

Zwerver, J., Bredeweg, S.W., \& Hof,A. L. (2007). Biomechanical analysis of the single-leg decline squat. British Journal of Sports Medicine, 41(4), 264-268; discussion 268. https: / / doi.org/ 10.1136/bjsm.2006.032482 